\title{
Biodiversité floristique et régénération naturelle sur les Hautes Terre de Lebialem (Ouest Cameroun)
}

\author{
Bertine TIOKENG ${ }^{1}$, Pierre-Marie MAPONGMETSEM ${ }^{1}$, \\ Victor François NGUETSOP ${ }^{2 *}$ et Walter Ndam TACHAM ${ }^{3}$ \\ ${ }^{1}$ Université de Ngaoundéré, BP 454 Ngaoundéré, Cameroun. \\ ${ }^{2}$ Université de Dschang, BP 67 Dschang, Cameroun. \\ ${ }^{3}$ Université de Bamenda, BP 39 Bambili, Bamenda, Cameroun. \\ *Auteur correspondant, E-mail: vfnguetsop@yahoo.fr
}

\section{RESUME}

Afin d'évaluer la diversité floristique, la structure du peuplement et la régénération naturelle, le présent travail a été mené sur les Hautes Terres de Lebialem dans la forêt de Bangang (Ouest Cameroun). Au total, 10 relevés floristiques de $250 \mathrm{~m}$ x $20 \mathrm{~m}$ ont été réalisés ; 6 sous parcelles de $100 \mathrm{~m}$ x 20 m ont été aussi établies dans 6 des 10 relevés. A l'intérieur de chaque relevé, tous les individus ayant un diamètre $\geq 10 \mathrm{~cm}$ à $1,30 \mathrm{~m}$ de hauteur ont été pris en compte. Pour les sous parcelles, les diamètres compris entre 2 et $9 \mathrm{~cm}$ à $0,2 \mathrm{~m}$ de hauteur ont été considérés. Dans l'ensemble, un total de 6972 individus appartenant à 191 espèces, 165 genres et 64 familles a été recensé. Napoleonaea egertonii Baker a la Valeur d'Importance d'espèce (IVI) la plus élevée. Les Fabaceae montrent une forte représentativité par rapport à leur valeur d'importance des familles. Les valeurs d'indice de diversité de Shannon Weaver (2,97 à 5,80 bits) traduisent une grande diversité spécifique du site ; l'Equitabilité de Piélou $(0,52$ à 0,96$)$ témoigne une bonne répartition des individus au sein des espèces. La densité des populations juvéniles indique un état de régénération appréciable (2239,16 tiges/ha) au sein du peuplement.

(c) 2015 International Formulae Group. All rights reserved.

Mots clés : Hautes Terres de Lebialem, biodiversité végétale, structure du peuplement, populations juvéniles, régénération naturelle, types phytogéographiques.

\section{INTRODUCTION}

Les écosystèmes forestiers sont extrêmement riches en espèces végétales et fauniques parfois peu ou mal connues. Cependant, l'évolution croissante du taux de déforestation actuel surtout dans les zones tropicales (FAO, 2001) risque entraîner l'érosion de certaines essences forestières non encore connues. En effet, l'absence d'informations quantitatives sur l'abondance et la distribution des organismes tropicaux demeurent jusqu'ici un problème pour la conservation de la biodiversité et gestion durable des écosystèmes forestiers. Du coup, il devient difficile de savoir l'espèce la plus menacée (Kenfack et al., 2006). De nombreux inventaires forestiers traditionnels ont souvent mis l'accent sur le potentiel d'exploitation du bois et ne fournissent donc que peu d'informations ou aucune sur les arbres de plus petite taille. Pourtant, la somme de savoirs accumulés est importante et la 
diversité interspécifique et intraspécifique des écosystèmes restent essentielles pour une conservation et une gestion durable des ressources forestières (Hunter, 1999).

Les forêts du Cameroun ont fait l'objet depuis longtemps de nombreux inventaires (Achoundong, 1996 ; Tchouto et al., 2006 ; Gonmadjé et al., 2011). Les Hautes Terres de Lebialem (Ouest Cameroun), considérées comme des sites de conservation prioritaire, ceci grâce à leur position de transition allant des forêts denses humides jusqu'à la végétation de savane en passant par les forêts de piedmont et submontagnardes (Harvey et al., 2004), sont encore peu explorées. Ces formations végétales qui font partie des forêts atlantiques biafréennes et des Hautes Terres, sont des centres d'endémisme et de biodiversité à l'échelle continentale (Bergl et al., 2007). Toutefois, les menaces globales telles que les changements climatiques, la destruction des forêts pour les besoins en terres cultivables et la cueillette de produits forestiers contribuent à une diminution de cette végétation naturelle, d'où le besoin urgent pour les actions de conservation et de protection.

Des études botaniques rapides récentes ont montré un nombre croisant d'espèces rares dans cet espace allant des montagnes de Bamenda au Nord jusqu'aux montagnes de Bakossi/Mwanenguba au Sud (Harvey et al., 2004). Mais, les données sur les forêts des Hautes Terres de Lebialem (Ouest Cameroun) restent encore très fragmentaires. Elles ne permettent pas d'avoir une vision continue de la flore du massif. On y note de rares travaux tels que l'étude des plantes médicinales utilisées en pharmacopée traditionnelle dans le village Aguambou-Bamumbu (Focho et al., 2009) et la publication d'une checklist de conservation établie à partir des inventaires dans les villages Fossimondi et Betchati (Harvey et al., 2010).

Le but de cette étude est d'évaluer la diversité floristique et de montrer la structure du peuplement; de déterminer les aires de distribution des espèces rencontrées dans le milieu étudié et d'examiner la régénération naturelle à travers les populations juvéniles de la végétation forestière du village Bangang.

\section{MATERIEL ET METHODES}

\section{Site d'étude et mise en place des parcelles d'inventaire}

Les Hautes Terres de Lebialem sont situées dans la région administrative du SudOuest Cameroun entre les versants Sud et Sud-Ouest des monts Bambouto. Ces derniers culminent à $2740 \mathrm{~m}$ entre le mont Oku (3011 $\mathrm{m})$ et le mont Cameroun (4095 m) sur les Hautes Terres de l'Ouest Cameroun qui couvrent une superficie de $1223 \mathrm{~km}^{2}$. Le climat du Lebialem est un climat équatorial de montagne. Il est caractérisé par des pluies très abondantes (3000 à $5000 \mathrm{~mm} / \mathrm{an}$ ) qui s'étalent sur une période de 10 à 12 mois dans l'année. Les températures varient entre $18^{\circ}$ et $20^{\circ} \mathrm{C}$ (Batadjomo et Götz, 2006). La géologie est constituée de granites, de roches volcaniques et sédimentaires. Les sols sont qualifiés d'andosols car caractérisés par l'abondance dans leur fraction minérale de produits amorphes silico-alumino-ferrugineux associés à des teneurs variables en matière organique ; ils ont aussi une texture généralement sableuse et à faible épaisseur (Sieffermann, 1973). Cette étude sera focalisée sur la formation forestière du village Bangang située à une altitude variant entre $200 \mathrm{~m}$ et $600 \mathrm{~m}$; dont les coordonnées moyens sont de $5^{\circ} 36^{\prime} 10,5^{\prime}$ ' de latitude Nord et de 9॰54'24,5', de longitude Est (Figure 1).

L'inventaire des espèces et le dénombrement de tous les individus d'un diamètre $\geq 10 \mathrm{~cm}$ à $1,30 \mathrm{~m}(\mathrm{dhp})$ ont été effectués sur des relevés rectangulaires de 250 m x 20 m (Tchiengué, 2004 ; Hakizimana et al., 2011) ; soit une superficie totale de 5 ha. Au total, 10 placettes ont été disposées dans la forêt en considérant le maximum d'hétérogénéité. A l'intérieur de 6 relevés choisis parmi les 10, des sous placettes de $2000 \mathrm{~m}^{2}$ (100 m x 20 m) ont été établies pour quantifier les arbustes et arbrisseaux de circonférences plus faibles (2 à $9 \mathrm{~cm}$ ) à 0,20 
$\mathrm{m}$ de hauteur (Maingi, 2006). Les herbacées, les lianes et les épiphytes n'ont pas été pris en compte dans le cadre de ce travail. Ainsi, l'inventaire des sous parcelles a porté sur un total de 1,2 ha. Les espèces non identifiées in situ ont été récoltées et identifiées plus tard à l'Herbier National du Cameroun à Yaoundé. La nomenclature taxonomique adoptée est la classification botanique phylogénétique des angiospermes (APG III, 2009).

\section{Analyse des données}

Les données issues des relevés floristiques ont permis de calculer les paramètres écologiques comme la fréquence relative, la dominance relative et la densité relative de chaque espèce. La somme des valeurs de ces paramètres a permis d'obtenir l'importance de chaque espèce par rapport aux autres espèces du site à travers l'IVI (Index Value Importance) (Curtis et Macintosh, 1951 ; Kent et Coker, 2003). Cet indice est fréquemment utilisé en forêt tropicale pour décrire l'importance écologique des espèces (Yao Adou et N'Guessan EK, 2005 ; Gonmadjé et al., 2011; Agbodjogbe, 2011). La valeur d'importance des familles (VIF) de Cottam et Curtis (1956) reprise par plusieurs auteurs (Yao Adou et N'Guessan, 2005; Gonmadjé et al., 2011) a été aussi utilisée pour apprécier le rôle de chaque famille dans la structuration du peuplement végétal. La distribution des types phytogéographiques a été montrée à partir des traits généraux retenus de la phytochorologie africaine (White, 1983). Les types suivants ont été attribués aux espèces: Pan-africaines et Paléotropicales, Guinéo-congolaise, Guinéenne supérieure et inférieure, Guinéenne inférieure, Camerounaise et Sud-Ouest Camerounaise. L'état de régénération naturelle a été évalué à partir l'IVI de tous les individus de diamètre compris entre 2 et $9 \mathrm{~cm}$ (Rajemison, 2010 ; Agbodjogbe, 2011). L'analyse consiste en la détermination des paramètres tels que la fréquence relative, la dominance relative et la densité relative qui permettent d'apprécier la composition floristique et la structure horizontale (Blanc, 1998). Cela permet de voir d'une part l'état des peuplements d'avenir, et d'autre part l'importance relative des différentes espèces présentes dans les milieux.

Les différents paramètres étudiés sont exprimés à travers les formules suivantes :

Fréquence relative $=$ (Fréquence de l'espèce i / somme des fréquences de toutes les espèces) x 100 ;

Densité relative $=$ (nombre total d'individus d'une espèce / nombre total de tous d'individus de toutes les espèces de la communauté) x 100 ;

Dominance relative $=$ (surface basale d'une espèce / Surface basale total) x 100 ;

Index Value Importance ou Importance des espèces $=$ Fréquence relative + Densité relative + dominance relative (pour l'espèce)

Valeur d'Importance des Familles = Fréquence relative d'une famille + Densité relative d'une famille + dominance relative d'une famille.

La diversité alpha (diversité- $\alpha$ ) ou richesse spécifique définie comme le nombre total d'espèces recensées dans un espace considéré et la diversité bêta (diversité- $\beta$ ) qui est une mesure quantitative de la diversité des communautés ont été adoptées pour cette étude (Nshimba, 2008 ; Bisby, 1995 ; Tchouto et al., 2006 ; Rajemison, 2010 ; Gonmadjé et al., 2011; Agbodjogbe, 2011). L'indice de Shannon Weaver ( $\left.\mathrm{H}^{\prime}\right)$ et l'Equitabilité de Piélou ont été choisis pour quantifier cette diversité.

- L'indice de Shannon Weaver est noté $H^{\prime}=-\sum_{i=1}^{s} \frac{N_{i}}{N} * \log _{2}\left(\frac{N_{i}}{N}\right)$, avec

$N i$ : Nombre d'individus d'une espèce donnée $\mathrm{i}, \mathrm{i}$ allant de 1 à s (nombre totale d'espèces).

$N$ : Nombre d'individus total.

$\log$ : logarithme décimal. L'équitabilité de

Piélou $E Q=\frac{H^{\prime}}{\log _{2} N_{0}}$

$\mathrm{N}_{0}$ : nombre total d'espèces

$\mathrm{H}^{\prime}$ : indice de Shannon Weaver 


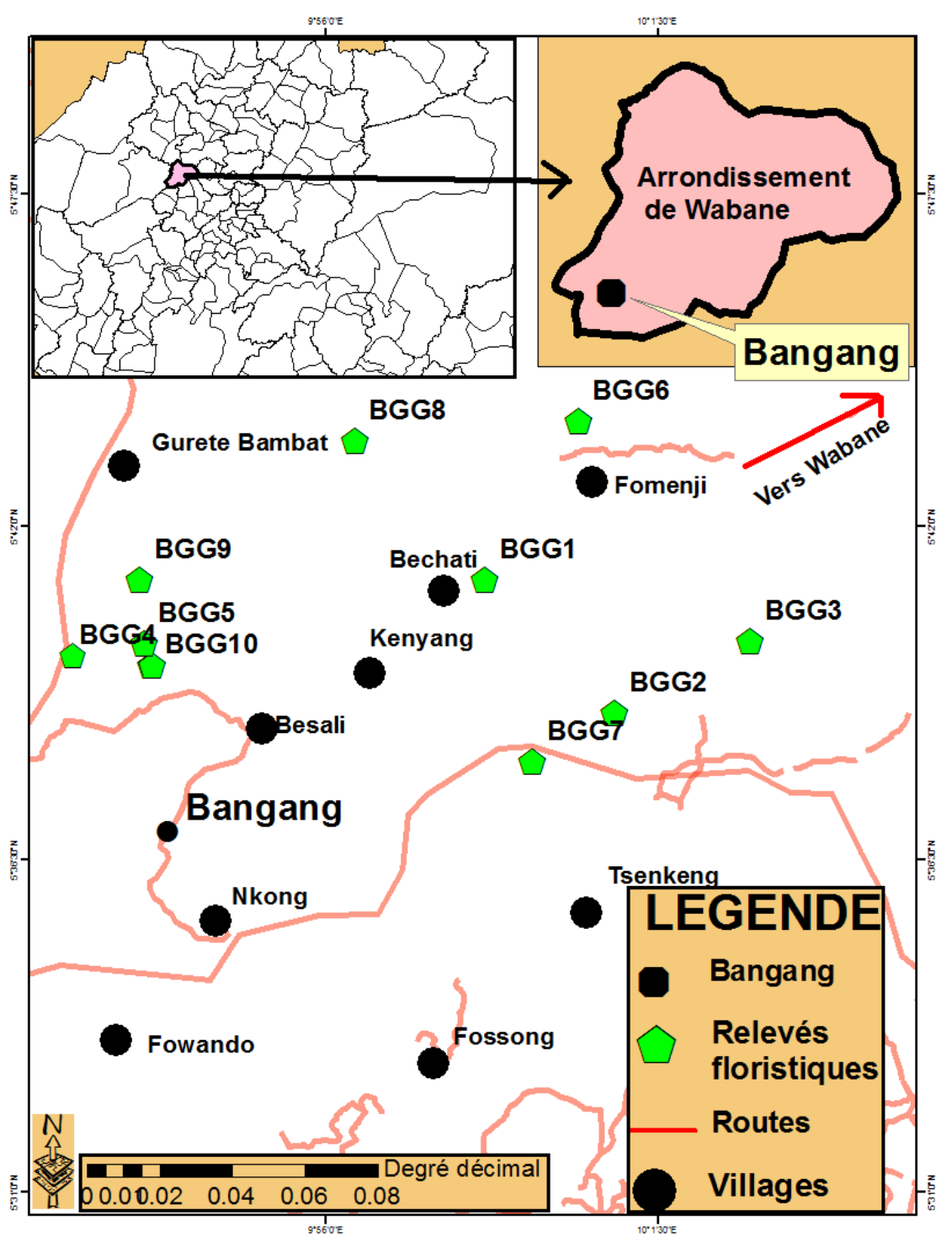

Figure 1: Situation du village Bangang dans l'Ouest Cameroun et localisation des relevés floristiques.

\section{RESULTATS}

Richesse spécifique et abondance des espèces en fonction de l'altitude

Un total de 4285 et 2687 tiges respectivement pour les individus de diamètres $\geq 10 \mathrm{~cm}$ et compris entre 2 et $9 \mathrm{~cm}$ ont été inventoriées dans des surfaces totales de 5 ha et 1,2 ha. L'ensemble de ces individus appartiennent à 191 espèces, 165 genres, 64 familles. Le nombre d'espèces varie entre 35 et 62 par relevé pour les tiges de diamètre $\geq 10 \mathrm{~cm}$ et entre 34 et 45 pour les tiges de diamètre compris entre 2 et $9 \mathrm{~cm}$.

De manière générale, les indices de diversité des individus de diamètre $\geq 10 \mathrm{~cm}$ calculée par relevé varient de 2,97 à 5,80 bits pour l'indice de diversité de Shannon et de 0,52 à 0,96 pour l'Equitabilité de Piélou. Le 
Tableau 1 montre que le nombre de tiges à l'hectare des individus de diamètres $\geq 10 \mathrm{~cm}$ varient entre 749 à 943 , le maximum étant atteint entre 300 et $400 \mathrm{~m}$ alors que le minimum est observé entre 400 et $600 \mathrm{~m}$ d'altitude. Les valeurs d'indices de diversité moyens par altitude montre que l'indice de Shannon $\left(\mathrm{H}^{\prime}=4,79\right)$ et l'Equitabilité de Piélou $(\mathrm{EQ}=0,85)$ sont maximales entre 400 et 600 $\mathrm{m}$. Les espèces les mieux représentées entre 200 et 300 sont Cola chlamydantha (176 individus), Anglylocalyx taboltii (146 individus) et Alexis cauliflora (140 individus). Entre 300-400 et 400-600 m, les espèces les plus abondantes sont respectivement Diogoa zenkeri (110 individus) et de Napoleonaea egertonii (157 individus).

D'après la phytogéographie africaine, les espèces guinéennes inférieures sont les plus représentées $(55,37 \%)$; ensuite viennent les espèces guinéo-congolaises $(30,57 \%)$ et les espèces qui sont à la fois guinéennes inférieures et congolaises $(16,52 \%)$. On note $4,13 \%$ d'espèces endémiques au Cameroun et $0,82 \%$ d'espèces à large distribution.

\section{Importance des espèces}

L'inventaire a permis de recenser 175 espèces de plante de diamètre $\geq 10 \mathrm{~cm}$ appartenant à 143 genres et 62 familles. Les espèces dont les valeurs d'indices d'importance (IVI) sont les plus élevées globalement (8 à 14,62) sont Treculia obbovoidea, Pycnanthus angolense, Anglylocalyx taboltii, Diogoa zenkeri (Engl.) Exell \& Andonça, Napoleonaea egertonii et Piptadeniastrum africana (Hook. f.) Brenan. Ces espèces prépondérantes figurent parmi les plus abondantes et /ou les plus dominantes (Tableau 2).

Les espèces les plus représentées en termes d'indice global d'importance entre 200 et $300 \mathrm{~m}$ sont Anglylocalyx taboltii (28,56\%), Alexis cauliflora $(18,99 \%)$, Cola pachycarpa $(11,38 \%)$. Entre 300 et 400 les plus fortes valeurs d'IVI sont observées chez Napoleonaea egertonii (26,11\%), Cola chlamydantha $(20,43 \%)$, Alexis cauliflora
$(20,35 \%)$. Entre 400 et 600 m, Napoleonaea egertonii (54,63\%), Treculia africana $(23,83 \%)$ et Pycnanthus angolensis $(21,08 \%)$ montrent les valeurs d'IVI les plus élevées.

\section{Importance des principales familles}

Les familles les plus importantes d'après la valeur d'importance des familles (VIF) (Tableau 3) représentent 32,65\% de l'ensemble des familles rencontrées; et $62,03 \%$ de tous les individus de diamètre $\geq 10$ $\mathrm{cm}$ recensés. Les cinq familles renfermant le plus grand nombre d'individus sont les Malvaceae (606 individus), les Fabaceae (323 individus), les Euphorbiaceae et Lecythidaceae (302 individus chacune) et les Olacaceae (282 individus). Les familles montrant un nombre d'espèces élevé (> 6) sont les Euphorbiceae (17 espèces), les Rubiaceae (17 espèces), les Malvaceae (14 espèces), les Fabaceae (6 espèces) et les Meliaceae (6 espèces).

Suivant l'altitude, la famille la plus prépondérante entre 200 et $300 \mathrm{~m}$ et entre 300 et $400 \mathrm{~m}$ est celle des Malvaceae $(61,39 \%$ et $38,69 \%$ respectivement). Par contre, entre 400 et $600 \mathrm{~m}$, la famille dominante est celle des Lecythydaceae $(53,91 \%)$.

\section{Evaluation de la régénération par graines}

Un total de 2687 individus de diamètre compris entre 2 et $9 \mathrm{~cm}$ a été recensé sur une superficie de 1,2 ha; soit une moyenne de 2239,16 tiges/ha. Ces individus appartiennent à 122 espèces, 103 genres et 51 familles. Parmi les 175 espèces rencontrées chez les individus de diamètre $\geq 10 \mathrm{~cm}, 108$ sont aussi présentes dans la population juvénile. Les familles les mieux représentées en termes d'individus sont les Menispermaceae (10,27\%), les Fabaceae $(9,64 \%)$, les Malvaceae $(9,23 \%)$, les Rubiaceae $(6,55 \%)$ et les Flacourtiaceae $(5,84 \%)$. Les cinq familles les plus riches en espèces sont les Rubiaceae (13 espèces), les Sterculiaceae (9 espèces), les Euphoriaceae (7 espèces), les Sapindaceae (6 espèces) et les Meliaceae (5 espèces). Angylocalyx oligophyllus renferme la plus 
grande valeur de l'IVI suivie de Alexis cauliflora et de Cola spp. (Figure 2).

Parmi les espèces à forte IVI (Figure 3 ), certaines renferment une densité absolue particulièrement élevée (comprise entre 78433 tiges). Il s'agit de : Alexis cauliflora (152 individus), Angylocalyx oligophyllus (433 individus), Cola spp. (187 individus), Homalium africanum (108 individus), Penianthus longifolus (784 individus),
Rinorea sp (93 individus) et Pleiocarpa rostrata (79 individus). La représentation des individus de régénération à forte abondance absolue et leurs individus de diamètre $\geq 10 \mathrm{~cm}$ en fonction des classes de diamètre révèle une distribution en exponentiel décroissante, avec un maximum dans la première classe de diamètre représentant les plantules; ensuite, celles des jeunes plants et en fin des individus semenciers.

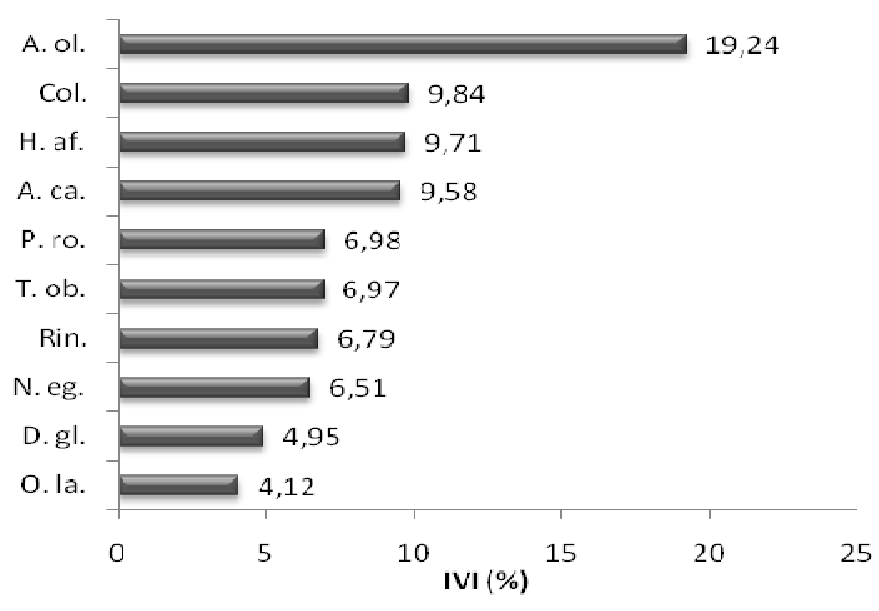

Figure 2: Valeur d'Importance de 10 espèces à forte régénération dans la forêt de Bangang, Ouest Cameroun. A. ol.: Angylocalyx oligophyllus; Rin. Rinorea sp; A. ca. : Alexis cauliflora; Treculia obovoidea; Col. : Cola spp.; D. gl. : Desbordesia glaucescens (Engl.) Van Tiegh; H. af. : Homalium africanum (Hook. f.) Benth; N. eg. : Napoleonaaea egertonii ; P. ro. : Pleiocarpa rostrata Stapf ; O. la. Olax latifolia Engl.

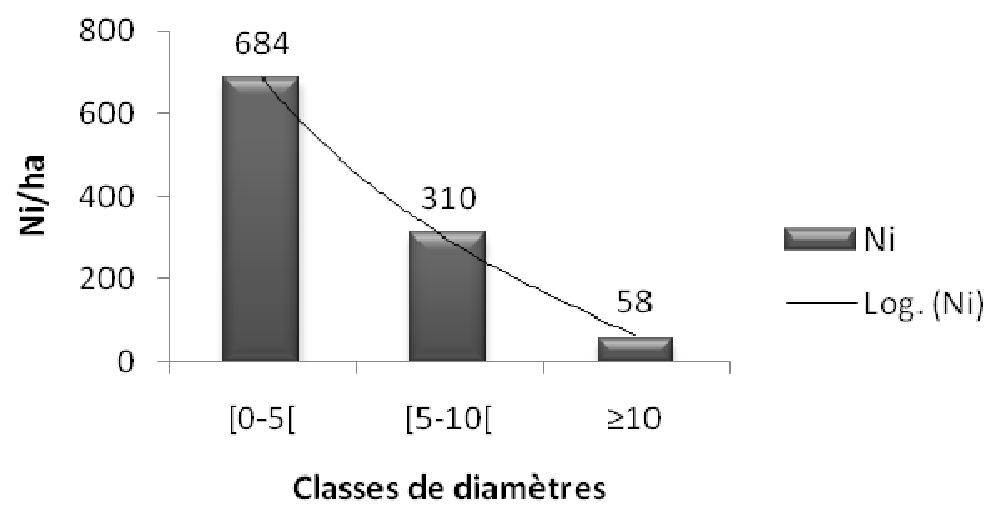

Figure 3: Répartition des individus des espèces à forte densité absolue (>78 tiges) en fonction des classes de diamètre sur une superficie de 1ha dans la forêt de Bangang, Ouest Cameroun. (Ni : Nombre d'individus). 
Tableau 1 : Nombre de relevés et leur superficie, caractérisation de la diversité floristique des individus à $\mathrm{dhp} \geq 10 \mathrm{~cm}$ dans la forêt de Bangang, Ouest Cameroun.

\begin{tabular}{|c|c|c|c|c|c|c|}
\hline $\begin{array}{l}\text { Altitudes } \\
(\mathrm{m})\end{array}$ & $\begin{array}{l}\text { Nombre de } \\
\text { relevés }\end{array}$ & $\begin{array}{l}\text { Superficie } \\
\text { (ha) }\end{array}$ & $\mathrm{Ne}$ & Ni.ha $^{-1}$ & $\mathrm{H}^{\prime}$ & EQ \\
\hline $200-300$ & 3 & 1,5 & 95 & 922 & 4,01 & 0,75 \\
\hline $300-400$ & 3 & 1,5 & 72 & 943 & 4,58 & 0,81 \\
\hline $400-600$ & 4 & 2 & 104 & 749 & 4,79 & 0,85 \\
\hline
\end{tabular}

Tableau 2 : Indice global d'importance des espèces prépondérantes (IVI> 2,77) dans la forêt de Bangang, Ouest Cameroun. Seuls les arbres à dhp supérieur ou égal à $10 \mathrm{~cm}$ sont considérés.

\begin{tabular}{lcccc}
\hline Espèces & $\mathbf{2 0 0 - 3 0 0}$ & $\mathbf{3 0 0 - 4 0 0}$ & $\mathbf{4 0 0 - 6 0 0}$ & IVI global \\
\hline Napoleonaea egertonii Baker f. & 9,67 & $\mathbf{2 6 , 1 1}$ & $\mathbf{5 4 , 6 3}$ & $\mathbf{1 4 , 6 2}$ \\
Alexis cauliflora (Oliv.) Pierre & $\mathbf{1 8 , 9 9}$ & $\mathbf{2 0 , 3 5}$ & 0,00 & 4,47 \\
Anglylocalyx taboltii Bak. Bak. f. & $\mathbf{2 8 , 5 6}$ & 3,97 & 14,69 & 9,03 \\
Canarium schweinfurthii Engl. & 3,49 & 0,00 & 10,99 & 4,53 \\
Chrysophylum albidum Engl. & 0,00 & 4,71 & 5,70 & 5,1 \\
Chytranthus cauliflorus Hutch \& Dalz & 11,05 & 5,64 & 4,25 & 4,76 \\
Cola chlamydantha (K. Schum.) Bodard & $\mathbf{2 3 , 7 6}$ & $\mathbf{2 0 , 4 3}$ & 0,00 & 6,98 \\
Cola hispida Brenan \& Keay & 6,96 & 0,00 & 8,82 & 4,1 \\
Cola pachycarpa K.Schum & 11,38 & 5,72 & 11,70 & 4,91 \\
Crotonogyne preussii pax. & 6,46 & 2,25 & 5,77 & 2,77 \\
Diogoa zenkeri (Engl.) Exell \& Andonça & 0,00 & $\mathbf{1 6 , 6 1}$ & $\mathbf{2 3 , 8 3}$ & 8,46 \\
Diospyros bipendensis Gürke & 0,00 & 7,31 & 7,33 & 3,24 \\
Erythrina senegalensis DC. & 1,12 & 2,96 & 9,94 & 3,79 \\
Garcinia manii Oliv. & 0,00 & 4,38 & 10,97 & 3,2 \\
Gouarea glomerulata Harms & 3,30 & 5,37 & 8,20 & 4,02 \\
Hymenostegia afzelii (Oliv.) Harms & 4,71 & 0,00 & 7,17 & 6,15 \\
Irvengia gabonensis (Aubrey-Lec.ex O'R.) Baill & 2,10 & 9,34 & 11,40 & 5,97 \\
Jollydora duparquetiana (Baill.) Pierre & 2,16 & 1,65 & 11,63 & 3,5 \\
Lophira alata Banks ex Gaerth & 10,78 & 0,00 & 1,90 & 4,63 \\
Massularia acuminata (G.Don) Bull.ex.Hoyle & 1,56 & 3,85 & 2,04 & 3,63 \\
Pentadesma grandifolia Bake. f. & 0,00 & 0,00 & 12,95 & 4,52 \\
Piptadeniastrum africana (Hook. f.) Brenan & 0,00 & 1,76 & 6,14 & 11,05 \\
Pycnanthus angolensis (Welw.) Warb. & 4,05 & 7,74 & $\mathbf{2 1 , 0 8}$ & 8,88 \\
Syzygium guineensis Engl. & 8,17 & 9,87 & 0,00 & 3,67 \\
Tapura africana Oliv. & 0,00 & 14,41 & 1,47 & 3,4 \\
Treculia africana Decne & 0,00 & 2,23 & 0,00 & 10,02 \\
Trichilia rubescens Oliv. & 7,32 & 2,23 & 6,30 & 4,08 \\
Uvariodendron giganteum (Engl.) R.E. Fries & 1,21 & 11,89 & 18,96 & 5,09 \\
\hline & & & & \\
\hline
\end{tabular}


Tableau 3: Indice d'importance des familles les plus représentées (VIF>6) dans la forêt de Bangang.

\begin{tabular}{lcccc}
\hline Familles & $\mathbf{2 0 0 - 3 0 0}$ & $\mathbf{3 0 0 - 4 0 0}$ & $\mathbf{4 0 0 - 6 0 0}$ & VIF global \\
\hline Malvaceae & 61,39 & 38,69 & 28,40 & 27,76 \\
Euphorbiaceae & 36,33 & 8,669 & 19,14 & 17,78 \\
Fabaceae & 37,52 & 21,35 & 20,76 & 29,61 \\
Meliaceae & 9,028 & 18,09 & 19,27 & 14,595 \\
Rubiaceae & 13,42 & 7,90 & 22,02 & 13,72 \\
Myristicaceae & 9,432 & 10,16 & 27,96 & 13,14 \\
Lecythydaceae & 16,16 & 25,51 & 53,91 & 12,03 \\
Moraceae & 9,66 & 34,69 & 29,13 & 12,01 \\
Olacaceae & 1,20 & 15,40 & 18,92 & 10,87 \\
Annonaceae & 5,61 & 16,51 & 10,49 & 9,32 \\
Violaceae & 20,76 & 19,91 & 17,56 & 8,57 \\
Lauraceae & 11,46 & 12,76 & 3,60 & 7,645 \\
Sapindaceae & 15,15 & 5,31 & 4,38 & 6,47 \\
Rutaceae & 6,00 & 0,77 & 0,00 & 6,26 \\
\hline
\end{tabular}

\section{DISCUSSION}

\section{Densité et diversité dans la forêt de Bangang}

La richesse spécifique des arbres à dhp $\geq 10 \mathrm{~cm}$ varie entre 48 et 72 espèces/ha. La variabilité de la richesse spécifique dans un peuplement dépendrait des conditions abiotiques et des interactions entre les espèces de chaque milieu et les différents biotopes. Les valeurs de la richesse spécifique de cette étude sont proches de celles observées par Sunderland et Balinga (2005) qui ont noté 77 espèces ligneuses/ha dans la végétation du parc national de Nouabalé-Ndoki (Congo). Par contre, elles sont inférieures aux résultats obtenus par Sunderland et al. (2004) dans la réserve de Takamanda dans la région du SudOuest Cameroun (100 espèces/ha); de Gonmadje et al. (2011) dans la forêt de Ngovayang dans le Sud Cameroun avec un nombre d'espèces compris entre 99 et 112 à l'hectare. Dans la réserve du Dja (Cameroun), Lejoly (1994) a obtenu une valeur de 92 espèces ligneuses/ha. De même, au Gabon, Balinga (2006) dans le parc national de Waka
(106 espèces/ha), Vroh Bi Tra et al. (2010) dans les forêts denses de la Réserve Azaguié au Sud-Est de la Côte d'Ivoire (98 espèces/ha), Maingi et Marsh (2006) dans les Galeries forestières de Tana au Kenya (101 espèces/ha) ont obtenu des valeurs bien supérieures à celles obtenues sur les Hautes Terres du Lebialem. La forte richesse spécifique dans ces formations végétales reflèterait une plus grande variabilité des niches et donc des facteurs biotiques et abiotiques favorables au développement d'un grand nombre d'espèces.

L'indice de diversité de Shannon obtenu dans la forêt de Bangang se situe autour de 4 bits (Tableau 1). Ces valeurs élevées témoignent de la richesse spécifique de la zone. En effet, les communautés forestières considérées comme étant riches d'après l'indice de Shannon ont une valeur de 3,5 bits (Kent et Coker, 1992). Ces résultats sont comparables à ceux de Gonmadje et al. (2011) qui montrent des valeurs comprises entre 3,90 et 4,12 bits et de Tchouto et al. (2006) dans la forêt humide de Campo- 
Ma'an avec des valeurs de 4,73 à 5,14 bits. Balinga (2006) et Sunderland et al. (2004) ont également trouvé des valeurs comparables de l'indice de diversité de Shannon (3,92 bits) dans les forêts des Monts de Cristal au Gabon. Cette forte diversité peut être liée au fait que ces forêts ne semblent pas avoir connues de perturbations majeures comme le montre aussi les valeurs de l'Equitabilité de Piélou ( 0,75 à 0,85). Ces valeurs rentrent en effet dans l'échelle des valeurs considérées comme étant optimales (0,6 à 0,8) par Odum (1976). Elles traduisent une bonne répartition des individus au sein des espèces. Comme observé par d'autres chercheurs dans les formations forestières d'Afrique centrale (Doucet, 2003 ; Kenfack et al., 2006 ; Tchouto et al., 2006 ; Gonmadje et al., 2011), les espèces guinéennes inférieures et guinéo-congolaises sont prédominantes dans la forêt de Bangang. La prédominance des espèces du domaine guinéen inférieur dans la forêt de Bangang serait liée à l'influence des facteurs climatiques particuliers tels que la qualité du vent que reçoit cette forêt. En effet, ce secteur phytogéographique est particulièrement soumis à la mousson atlantique ainsi que, dans sa partie Sud, à l'effet refroidissant du courant du Benguela qui assure une humidité atmosphérique importante même en saison sèche (Suchel, 1972; White, 1983). La proportion élevée des espèces du domaine guinéo-congolais traduirait une grande maturité de cette forêt qui semble peu ou pas perturbée par l'activité humaine.

L'abondance des arbres de diamètre à hauteur de poitrine $\geq 10 \mathrm{~cm}$ oscille entre 749 et 943 tiges/ha dans les relevés étudiés. Lorsqu'on compare ces densités à celles des autres massifs forestiers, on constate qu'elles sont supérieures à celles trouvées dans d'autres formations forestières au Cameroun et notamment dans les forêts denses de la réserves du Dja avec 461 tiges/ha (Sonké et
Lejoly, 1998), dans la forêt de Ngovayang qui montre une valeur moyenne de 532 tiges/ha (Gonmadje et al., 2011). Les galeries forestières au Niger montrent aussi des valeurs inférieures à celles observées dans les relevés de Bangang (234-464 tiges/ha) d'après les travaux d'Agbodjogbe (2011), de même que les forêts du parc national de Waka (Gabon) où Balinga (2006) a montré une valeur moyenne de 589 tiges/ha. Par ailleurs, ces valeurs sont largement inférieures à celles de Vroh Bi Tra et al. (2010) avec 1173 pieds/ha dans les forêts denses de la Réserve Azaguié (Côte d'ivoire). Ces différences de densité seraient dues aux facteurs (exemple: sols, précipitations, compétition) qui déterminent la diversité et la structuration de la végétation en relation avec les paramètres biotiques. Les valeurs relativement élevées de la densité sur pieds peuvent être aussi expliquées par une influence anthropique qui est faible. En effet, ces forêts n'ont presque pas subi d'exploitation de grande importance. Les seules activités humaines notées se limitent pour le moment aux prélèvements du bois de feu ou de quelques espèces à caractère médicinal et d'autres produits forestiers non ligneux.

L'analyse de l'Indice d'importance des familles (VIF) a révélé une forte représentativité des Fabaceae. Ce résultat corrobore avec les travaux de Letouzey (1985) et Gonmadje et al. (2011) dans d'autres forêts en Afrique tropicale. L'abondance de cette famille dans une forêt indique que celle-ci appartient au type phytogéographique GuineoCongolais (White, 1983; Gonmadje et al., 2012). Cette famille est aussi considérée comme celle caractérisant les forêts anciennes (Leal, 2001). En outre, certaines familles telles que les Olacaceae et les Burseraceae figurent parmi les plus importantes. Elles sont considérées comme les indicatrices des forêts atlantiques sempervirentes (Doumenge, 1992 ; 
Senterre et Lejoly, 2001). Selon Doucet (2003), dans les forêts denses humides du Gabon, le niveau élevé d'endémisme est lié à la forte dominance des Fabaceae, Olacaceae et Burseraceae.

\section{Régénération naturelle}

La structure horizontale des individus de diamètre compris entre 2 et $9 \mathrm{~cm}$ montre que la régénération est effective dans la forêt de Bangang. Elle est observée chez 108 espèces sur un total de 175 espèces enregistrées pour les individus de diamètre $\geq$ $10 \mathrm{~cm}$. On note parmi celles-ci certaines qui n'ont pas été observées lorsque les diamètres des individus $\geq 10 \mathrm{~cm}$ ont été pris en compte. On peut penser que les jeunes tiges et arbustes offrent l'opportunité de rencontrer non seulement certaines espèces difficilement identifiables à l'état de gros arbres mais aussi celles dont les semenciers ne sont plus fortement représentées dans la forêt. La densité absolue moyenne des régénérations qui est de 2239,16 tiges /ha traduirait une régénération appréciable au sein des peuplements. Cette valeur est supérieure à celle trouvée par Agbodjogbe (2011) dans les galeries forestières de la réserve totale de Faune de Tamou (Niger) avec 1207 tiges/ha. Cette régénération est confirmée par la répartition des individus à forte densité absolue par classe de diamètre qui suit l'allure d'une formation végétale possédant une possibilité de renouvellement du peuplement. Les observations similaires ont été faites par Hakizimana et al. (2011). D'après Bouko et al. (2007), une telle distribution diamétrique traduirait un état d'équilibre dans un milieu, ce qui implique une bonne régénération naturelle (Puig, 2001).

\section{Conclusion}

La diversité, la structure et la régénération naturelle du massif forestier de
Bangang dans les Hautes Terres de Lebialem ont été évaluées. C'est une forêt très diversifiée, renfermant également des espèces endémiques au Cameroun, à l'exemple de Medusandra mpomiana et Crotonogyne impedita. Parmi les espèces les plus abondantes on a: Napoleonaea egertonii, Diogoa zenkeri, Alexis cauliflora, Angylocalyx oligophyllus. La richesse spécifique et la densité des arbres à l'hectare sont proches des résultats trouvés par d'autres chercheurs. Les Fabaceae et les Malvaceae ont les valeurs d'importances les plus élevées. L'analyse des individus de diamètre compris entre 2 et $9 \mathrm{~cm}$ montre que la régénération naturelle dans la forêt de Bangang est effective et bonne de manière générale. L'image de la forêt que donne le premier niveau d'échantillonnage (4285 tiges de diamètre $\geq$ à $10 \mathrm{~cm}$ réparties sur 175 espèces pour une superficie totale de 5 ha échantillonnée) diffère de celle du second niveau d'échantillonnage (2687 individus de diamètre variant entre 2 et $9 \mathrm{~cm}$ repartis sur 122 espèces pour une superficie de 1,2 ha échantillonnée) notamment en termes de densité et du nombre d'espèces par hectare. La représentativité respective des familles change, par exemple, les Malvaceae ont un nombre plus important (606 tiges de diamètre $\geq 10 \mathrm{~cm}$ ) alors que pour les individus de diamètre compris entre 2 et $9 \mathrm{~cm}$, on note plutôt les Menispermaceae (276 tiges). Certaines espèces n'apparaissent que pour des tiges de gros diamètres et disparaissent quand le petit diamètre est pris en compte et vice versa, d'où l'intérêt que l'on peut avoir à définir les groupements végétaux en prenant en compte aussi des espèces de plus petits diamètres. Cependant, les fortes pressions anthropiques croissantes exercées sur les forêts tropicales en général, risqueraient conduire à une érosion irréversible des espèces de la forêt de Bangang encore très peu 
connue. Il serait important de faire des études approfondies sur tous ses contours aussi bien sur la diversité des plantes du massif que sur celle des animaux car cette forêt renfermerait en son sein des espèces endémiques menacées de disparition.

\section{REFERENCES}

Achoundong G. 1996. Les forêts sommitales au Cameroun. Bois et Forêts des Tropiques, $247: 8$.

Agbodjogbe GJ. 2011. Analyse de la Structure des Galeries Forestières de la Réserve Totale de Faune de Tamou (RTFT) en République du Niger. Thèse de Master international, Muséum national d'histoire naturelle, Paris, IRD, Sud expert plantes, Université de Dschang, Université Abdou Moumouni, 59 p.

APG III. 2009. An update of the Angiosperm Phylogeny Group classification for the orders and families of flowering plants: APG III. In Botanical Journal of the Linnean Society, 161(2): 105-121.

Balinga MPB. 2006. A vegetation assessment of the Waka national park, Gabon. CARPE Report. http://carpe.umd.edu/ resources/Documents/. Cited 4 July 2009

Batadjomo BG, Götz E. 2006. Rapport de fin de mission d'appui technique au classement des unité Technique Opérationnelle de Takamanda-mone, de Korup- ndongere et au changement de statuts des réserves forestières de Takamanda et Bomboko en parcs Nationaux. PGDRN,Gtz, Sud-Ouest Cameroun, $152 \mathrm{p}$.

Bergl RA, Oates JF, Fotso R. 2007. Distribution and protected area coverage of endemic taxa in West Africa's Biafran Forests and Highlands. Biological Conservation, 134: 195-208.

Bisby FA. 1995. Characterization of biodiversity. In Global Biodiversity
Assessment, Heywood VH, Watson RT (eds). UNEP; Cambridge University Press: Cambridge; 192-294.

Blanc L. 1998. Les formations forestières du Parc National de Cat Tien (Viêt-Nam) : caractérisation structurale et floristique, étude de la régénération naturelle et de la dynamique successionnelle. Thèse de doctorat, Université Claude Bernard, Lyon 1, 207 p.

Bouko SB, Sinsin B, Soulé GB. 2007. Effets de la dynamique d'occupation du sol sur la structure et la diversité des forêts claires et savanes du Bénin. Tropicultura, 25(4): 221-227.

Cottam G, Curtis JT. 1956. The use of distance measures in phytosociological sampling. Ecology, 37: 451-460.

Curtis JT, McIntosh RP. 1951. An upland forest continuum in the prairie-forest border region of Wisconsin. Ecology, 32: 476-496.

Doucet JL. 2003. L'alliance délicate de la gestion forestière et de la biodiversité dans les forêts du centre du Gabon. Thèse de Doctorat, Faculté universitaire des sciences agronomiques de Gembloux, Belgique, $283 \mathrm{p}$.

Doumenge C. 1992. La réserve de Conkouati: Congo, le Secteur Sud-Ouest. UICN : Chevron, Gland-Suisse ; 213.

FAO. 2001. Situation des Forêts dans le Monde. FAO: Rome; 181.

Focho DA, Ndam WT, Fonge BA. 2009. Medicinal plants of Aguambu -Bamumbu in the Lebialem highlands, Southwest province of Cameroon. African Journal of Pharmacy and Pharmacology, 3(1): 001-013.

Gonmadje F, Doumenge C, Mckey D, Tchouto GPM, Sunderland TCH, Balinga MPB, Sonké B. 2011. Tree diversity and conservation value of Ngovayang's 
lowland forests, Cameroon. Biodiversity and Conservation, 20(12): 2627-2648.

Gonmadje CF, Doumenge C, Sunderland CHT, Balinga PBM, Sonké B. 2012. Analyse phytogéographique des forêts d'Afrique Centrale: le cas du massif de Ngovayang (Cameroun). Plant Ecology and Evolution, 145(2): 152-164.

Hakizimana P, Bangirinama F, Havyarimana F, Habonimana B, Bogaert J. 2011. Analyse de l'effet de la structure spatiale des arbres sur la régénération naturelle de la forêt claire de Rumonge au Burundi. Bull. Sci. Inst. Nat. Environ. Conserv. Nat., 9: 46-52.

Harvey Y, Pollard BJ, Darbyshire I, Onana JM, Cheek M. 2004. The plants of Bali Ngemba Forest Reserve, Cameroon: A Conservation Checklist. Royal Botanic Gardens: Kew, UK; IV+154.

Harvey Y, Tchiengué B, Cheek M. 2010. The Plants of Lebialem Highlands, Cameroon: A Conservation Checklist. Royal Botanic Gardens: Kew; 170 .

Hunter ML. 1999. Maintaining Biodiversity in Forest Ecosystems. Cambridge University Press: Cambridge; xiv +698 .

Kenfack D, Thomas DW, Chuyong G, Condit R. 2006. Rarity and abundance in a diverse African forest. Biodivers Conserv. doi:10.1007/s10531-006-90652. $28 \mathrm{p}$.

Kent M, Coker P. 2003. Vegetation Description and Analysis - a Practical Approach. John Wiley \& Son Eds: UK; 354p. + annexes.

Leal ME. 2001. Microrefugia, small scale ice age forest remnants. In Plant Systematics and Phytogeography for the Understanding of African Biodiversity, Robbrecht E, Degreef J, Friis I (eds). Proceedings XVIth AETFAT congress. Systematics and Geography of Plants (vol
71). National Botanic Garden of Belgium : Meise ; 1073-1077.

Lejoly J. 1994. Mise en place des travaux en vue des inventaires de biodiversité dans la forêt de Ngotoo (République Centrafricaine). Projet Ecofac, AgrecoCTFT, Bruxelles, 109p.

Letouzey R. 1985. Notice de la Carte Phytogéographique du Cameroun au 1/500000. IRA Yaoundé, Inst. Cart. Intern. Végétation : Toulouse ; 240.

Maingi JK, Marsh SE. 2006. Composition, structure, and regeneration patterns in a gallery forest along the Tana River near Bura, Kenya. Forest Ecology and Management, 236: 211-228.

Nshimba H. 2008. Etude foristique, écologique et phytosociologique des forêts de l'île Mbiye à Kisangani, RD Congo. Thèse de Doctorat, Université Libre Bruxelle, 272 p.

Odum EP.1976. Écologie. Doin. Paris ; 257 p. Puig H. 2001. La Forêt Tropicale Humide. Editions Berlin : Paris ; 448

Rajemison AH. 2010. Typologie de la végétation rémanente en vue d'une restauration écologique. Cas du site d'Ankafobe-Tampoketsa d'AnkazobeHautes terres centrales de Madagascar. Memoire DEA, université d'Antananarivo, 83 p.

Senterre B, Lejoly J. 2001. Tree diversity in the Nsork rain forest (Rio Muni, Equatorial Guinea). Acta Bot Gallica, 148: $227-235$.

Sieffermann G. 1973. Les sols de quelques régions volcaniques $d u$ Cameroun. Mémoire ORSTOM. Edition de l'office de la Recherche Scientifique et Technique Outre-Mer, 179p.

Sonké B, Lejoly J. 1998. Biodiversity study in Dja fauna reserve (Cameroon): using the transect method. In Chorology, Taxonomy and Ecology of the Floras of 
Africa and Madagascar, Huxley CR, Lock JM, Cutler DF (eds). Royal Botanic Gardens: Kew; 171-179.

Suchel JB. 1972. La Répartition des Pluies et Régimes Pluviométriques au Cameroun. Contribution à l'Etude des Climats de l'Afrique Tropicale. Travaux et Documents de Géographie Tropicale. 5 Univ. Féd. Cam, Ed. CEGET-CNRS : Bordeaux, France; 287 p.

Sunderland TCH, Balinga MB. 2005. Evaluation préliminaire de la végétation du parc national de Nouabale-Ndoki et de sa zone tampon, Congo. CARPE Report. http://carpe.umd.edu/resources/Document s/.

Sunderland TCH, Walters G, Issembe Y. 2004. A preliminary vegetation assessment of the Mbé National Park, Monts de Cristal, Gabon. CARPE Report. http://carpe.umd.edu/resources/Document s/.

Tchiengue B. 2004. Etude écologique et floristique de la végétation d'un massif de la ligne du Cameroun : le Mont Koupe, Thèse de Doctorat $3^{\mathrm{e}}$ cycle. 238 p.

Tchouto GP, Yemefack M, De Boer WF, De Wilde JJFE, Cleef AM. 2006. Biodiversity hotspots and conservation priorities in the Campo-Ma'an rainforests, Cameroon. Biodivers Conserv, 15: 1219-1252.

Vroh Bi Tra AA, Kouame Y, N'da D, N'guessan K. 2010. Diversité Floristique et Structurale sur le Site d'une Réserve Naturelle Volontaire à Azaguié, Sud-est de la Côte d'Ivoire. European Journal of Scientific Research, 45(3): 411-421.

White F. 1983. The Vegetation of Africa. A Descriptive Memoir to Accompany the UNESCO/AETFAT/UNSO Vegetation Map of Africa. Natural Resources Research, 20, UNESCO: Paris.

Yao Adou CY, N'Guessan EK. 2005. Diversité botanique dans le sud du parc national de Taï, Côte d'Ivoire. Afrique Science, 01(2): 295 - 313. 